\title{
OPEN Role of 11ß-hydroxysteroid dehydrogenase type 1 in the development of atopic dermatitis
}

\author{
Noo Ri Lee ${ }^{1}$, Beom Jun Kim ${ }^{1}$, Chung Hyeok Lee ${ }^{1}$, Young Bin Lee ${ }^{1}$, Solam Lee ${ }^{1}$, \\ Hyun Jee Hwang ${ }^{1}$, Eunjung Kim ${ }^{1}$, Sung Hee Kim², Min-Geol Lee², Sang Eun Lee ${ }^{3}$, \\ Gareth G. Lavery ${ }^{4}$ \& Eung Ho Choi ${ }^{1 凶}$
}

Glucocorticoids (GCs) are potent anti-inflammatory drugs, the secretion of which is mediated and controlled by the hypothalamic-pituitary-adrenal axis. However, they are also secreted de novo by peripheral tissues for local use. Several tissues express $11 \beta$-hydroxysteroid dehydrogenase 1 (11 $\beta$ HSD1), including the skin. The inactive GC cortisone is converted by $11 \beta-H S D 1$ to active GC cortisol, which is responsible for delayed wound healing during a systemic excess of GC. However, the role of $11 \beta$-HSD1 in inflammation is unclear. We assessed whether 11ß-HSD1 affects the development of atopic dermatitis (AD) in vitro and in vivo. The expression of $11 \beta$-HSD1 in the epidermis of AD lesions was higher than that in the epidermis of healthy controls. Knockdown of 11ß-HSD1 in human epidermal keratinocytes increased the production of thymic stromal lymphopoietin. In an oxazoloneinduced mouse model of AD, localized inhibition of 11 $\beta$-HSD1 aggravated the development of AD and increased serum cytokine levels associated with AD. Mice with whole-body knockout (KO) of $11 \beta$-HSD1 developed significantly worse AD upon induction by oxazolone. We propose that $11 \beta-H S D 1$ is a major factor affecting $A D$ pathophysiology via suppression of atopic inflammation due to the modulation of active GC in the skin.

Cortisol is an endogenous glucocorticoid (GC) in humans, whose release is stimulated by the activation of the hypothalamic-pituitary-adrenal (HPA) axis by various stressors ${ }^{1}$. Exogenous GCs have been used as a potent anti-inflammatory drug for chronic inflammatory diseases, including atopic dermatitis $(\mathrm{AD})^{2,3}$. The main antiinflammatory mechanism of GCs is the suppression of inflammatory gene transcription factors such as nuclear factor-kappa B (NF- $\kappa \mathrm{B})$ and activator protein- $1^{4}$.

Apart from systemic GC production elicited by the HPA axis, extra-adrenal GCs are synthesized de novo in various tissues, including the brain, colon, heart, lung, thymus, and $\operatorname{skin}^{5-9}$. Epidermal and follicular keratinocytes, melanocytes, and dermal fibroblasts in the skin have glucocorticosteroidogenic activity ${ }^{5,6,10-13}$.

$11 \beta$-Hydroxysteroid dehydrogenases (11 $\beta$-HSDs) regulate the peripheral availability of GCs. There are two isoforms of $11 \beta$-HSD: $11 \beta$-HSD1 converts inactive cortisone into cortisol ${ }^{14,15}$, whereas $11 \beta$-HSD2 inactivates cortisol to cortisone ${ }^{16,17}$. Epidermal keratinocytes, dermal fibroblasts, sebocytes, and sweat gland cells in the skin, and the outer root sheath cells of hair follicles express $11 \beta$-HSD1 $1^{7,18-20}$, and this expression is increased by ultraviolet (UV) B and UVC but not UVA irradiation ${ }^{17,21}$. Increased $11 \beta-H S D 1$ expression leads to increased availability of local GCs and increased suppression of inflammatory responses in keratinocytes ${ }^{22}$. The expression of $11 \beta$-HSD1 mRNA was reportedly higher in human dermal fibroblasts (HDFs) obtained from a donor-matched photo-exposed outer upper arm than in HDFs obtained from the photo-protected inner upper arm ${ }^{19}$. The expression and activity of $11 \beta$-HSD1 were elevated in biopsies of photo-exposed skin (outer lower arm) compared to those in biopsies of donor-matched photo-protected skin (inner upper arm) in vivo ${ }^{23}$. Inflammation in response

\footnotetext{
${ }^{1}$ Department of Dermatology, Yonsei University Wonju College of Medicine, Wonju, Republic of Korea. ${ }^{2}$ Department of Dermatology, Severance Hospital, Cutaneous Biology Research Institute, Yonsei University College of Medicine, Seoul, Republic of Korea. ${ }^{3}$ Department of Dermatology, Cutaneous Biology Research Institute, Yonsei University College of Medicine, Gangnam Severance Hospital, Seoul, Republic of Korea. ${ }^{4}$ Institute of Metabolism and Systems Research, College of Medical and Dental Sciences, University of Birmingham, Birmingham B15 2TT, UK.『email: choieh@yonsei.ac.kr
} 
(a)

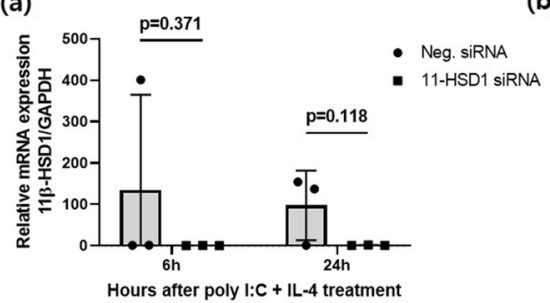

(b)

6h incubation after poly I:C + IL-4 treatment

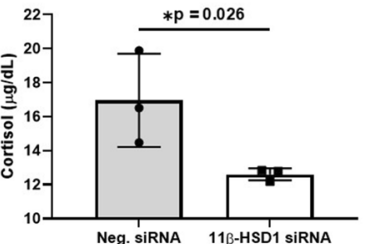

(c)

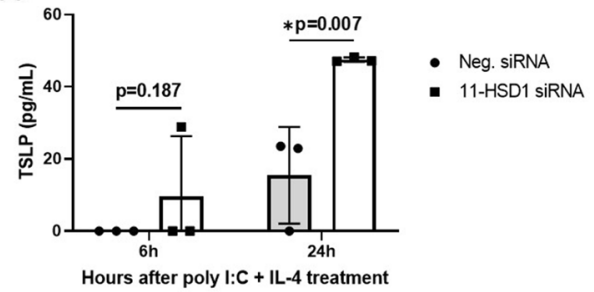

(d)

24h incubation after poly I:C + IL-4 treatment

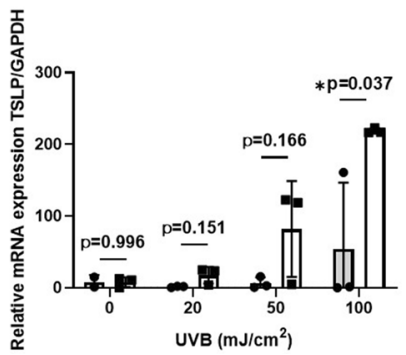

(e)

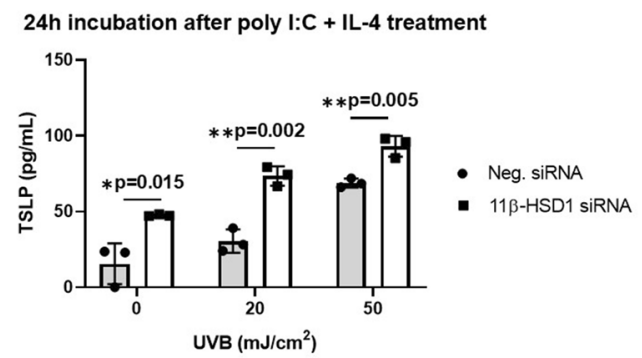

Figure 1. Effect of 11 $\beta$-HSD1 knockdown on TSLP production in NHEK cells. (a) We transfected NHEK with either $11 \beta$-HSD1 siRNA or negative siRNA then mRNA expression of $11 \beta-H S D 1$ was quantified by qPCR after six and $24 \mathrm{~h}$ incubations with poly I:C and IL-4. (b) Cortisol in culture medium measured after six-hour incubation with poly I:C and IL-4. (c) Amount of TSLP in culture media measured after six and $24 \mathrm{~h}$ incubations with poly I:C and IL-4. (d) mRNA expression of TSLP quantified by qPCR after irradiation with $0,20,50,100 \mathrm{~mJ} / \mathrm{cm}^{2}$ UVB. (e) Amount of TSLP measured after irradiation with 0,20 and $50 \mathrm{~mJ} / \mathrm{cm}^{2}$ $\mathrm{UVB}\left({ }^{*} p<0.05,{ }^{* *} p<0.005\right)$. Data are presented as means \pm standard deviation. IL-4, interleukin-4; poly I:C, polyinosinic:polycytidylic acid; siRNA, small interfering RNA; UVB, ultraviolet $B$.

to hapten-induced dermatitis was exacerbated and the response to topical 11-dehydrocorticosterone was attenuated in keratinocyte-specific $11 \beta$-HSD1 (HSD11b1) knockout $(\mathrm{KO})$ mice compared with the reactions in WT mice. These findings confirmed that GCs exert their immunosuppressive effect through $11 \beta-\mathrm{HSD}^{24}$. Therefore, $11 \beta$-HSD1 is not only involved in the cutaneous side effects caused by excess GCs but also plays a key role in local anti-inflammatory activity in keratinocytes.

$\mathrm{AD}$ is one of the most common inflammatory diseases of the skin. It is characterized by severe pruritus and its multifactorial pathogenesis involves a complex combination of epidermal barrier defects, T helper cell type 2 (Th2)-skewed inflammation, and neuroendocrinal dysregulation ${ }^{14,25-28}$. The involvement of GCs in inflammatory skin diseases such as AD has been proposed recently. Several studies have described that patients with AD show a blunted response to psychological stress with lower cortisol production compared with normal controls ${ }^{29-31}$. In mice, the absence of GC receptors in the epidermis results in skin barrier defects and increased susceptibility to cutaneous inflammation $^{32}$. The role of $11 \beta$-HSD1 in AD has not been studied yet. However, evidence from recent studies supports the notions that $11 \beta-H S D 1$ is involved in inflammatory cytokine expression in keratinocytes and that it plays an important role in AD exacerbation by modulating local GC availability ${ }^{14}$. The expression of thymic stromal lymphopoietin (TSLP) is significantly increased in the epidermis of keratinocyte-specific Hsd11b1 KO compared to that in WT mice ${ }^{33}$. Moreover, GCs suppress the upregulation of TSLP in normal human epidermal keratinocytes (NHEK) transfected with 11 $\beta$-HSD1 small interfering RNA (siRNA). Thus, endogenous GCs activated by the homeostatic activation of $11 \beta$-HSD1 might regulate TSLP production ${ }^{33}$.

In this study, we aimed to determine the impact of regulation of endogenous GCs by $11 \beta$-HSD1 on the development of $\mathrm{AD}$ in vitro and in vivo.

\section{Results}

Knockdown of 11ß-HSD1 by siRNA transfection increases TSLP production in NHEKs. After 6 and $24 \mathrm{~h}$ of poly I:C and IL- 4 treatment, the mRNA expression level of $11 \beta$-HSD 1 tended to decrease in siRNA-transfected NHEKs (Fig. 1a). Moreover, the cortisol concentration in the culture medium of siRNAtransfected cells was significantly lower after $6 \mathrm{~h}$ of treatment (Fig. 1b). Therefore, 11 $\beta$-HSD1 siRNA transfection knocked down $11 \beta$-HSD1 expression and $11 \beta$-HSD1 was responsible for the regulation of cortisol production. Both $6 \mathrm{~h}$ and $24 \mathrm{~h}$ after incubation, stimulation of TSLP production by polyinosinic:polycytidylic acid (poly I:C) and interleukin-4 (IL-4)levels were higher in siRNA-transfected cells (Fig. 1c). UVB irradiation induced TSLP expression in human keratinocytes via stabilization of hypoxia-inducible factor-1a (HIF-1 $\alpha)^{34}$. The expression of TSLP mRNA was increased in siRNA-transfected NHEKs, and the difference was significant at $100 \mathrm{~mJ} / \mathrm{cm}^{2}$ UVB (Fig. 1d). TSLP levels produced by siRNA-transfected cells were significantly higher than those produced by the control cells after irradiation with 0,20 , and $50 \mathrm{~mJ} / \mathrm{cm}^{2} \mathrm{UVB}$ (Fig. 1e). 
(a)

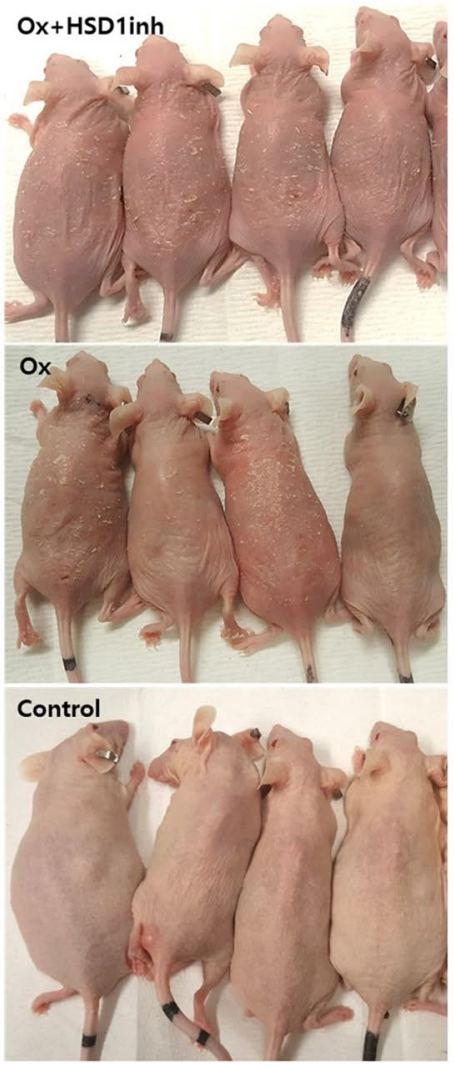

(b)

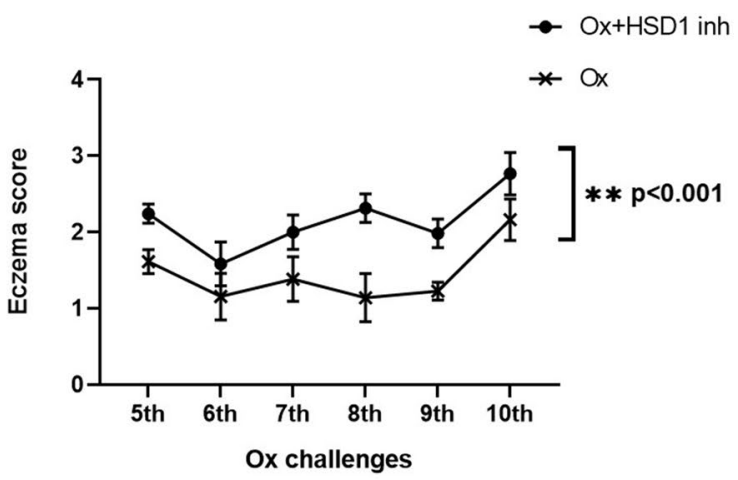

(c)

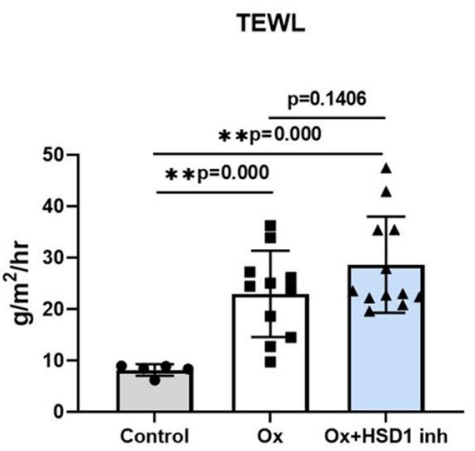

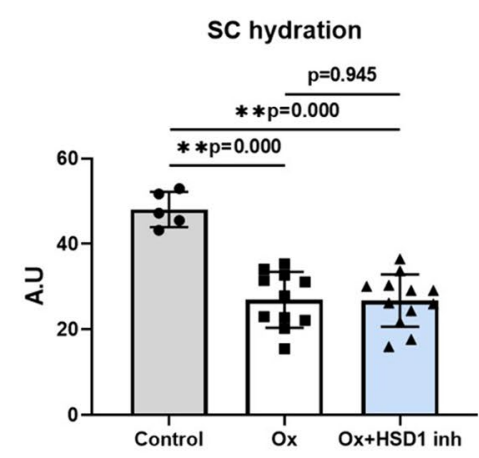

Figure 2. Effect of topical selective $11 \beta$-HSD1 inhibitor on oxazolone-evoked AD. (a) Gross photograph of control $(n=5)$, and Ox-induced female hairless $(\mathrm{hr} / \mathrm{hr})$ mice with topically applied vehicle $(\mathrm{n}=7)$ and $11 \beta$-HSD1 inhibitor $(n=7)$ taken before sacrifice (at 26 days). (b) Eczema scores of Ox-induced mice treated with $11 \beta$ HSD1 inhibitor and vehicle at challenges 5-10. (c) TEWL and SC hydration in control and Ox-induced mice with vehicle and $11 \beta$-HSD1 inhibitor before sacrifice (at 26 days) $\left({ }^{*} p<0.05,{ }^{* *} p<0.005\right)$. Data are presented as means \pm standard deviation. HSD1 inh $11 \beta$-HSD1 inhibitor, $O x$ oxazolone, SC stratum corneum, TEWL transepidermal water loss.

Topical application of selective 11ß-HSD1 inhibitor aggravates phenotypes inoxazolone-treated mice. Oxazolone (Ox)-treated mice developed typical AD lesions. Mice treated topically with $11 \beta$-HSD1 inhibitor displayed worse eczema lesions than the vehicle-treated group (Fig. 2a). The eczema score of the $11 \beta$-HSD1 inhibitor-treated group was significantly higher than that of the vehicle-treated group (Fig. 2b). Transepidermal water loss (TEWL) of both Ox-AD mouse groups was significantly increased compared to that of the control group. The 11 $\beta$-HSD1 inhibitor-treated group displayed a higher (but not statistically significant) TEWL than the vehicle-treated group. SC hydration of Ox-treated mice was significantly lower than that of control mice, but there was no difference between the vehicle- and the 11 $\beta$-HSD1 inhibitor-treated groups (Fig. 2c).

Serum inflammatory cytokines tend to increase in Ox-AD mice. We measured the levels of various inflammatory cytokines in mouse sera using ELISA (Fig. 3). Tumor necrosis factor-alpha (TNFa) level was increased in the Ox-AD group compared to that in the control group, but there was no difference between the $11 \beta$-HSD1 inhibitor-treated Ox-AD group and the vehicle-treated Ox-AD group. Serum IL-10 levels of the Ox-AD groups were higher than those of the control mice, and mice treated with 11 $\beta$-HSD1 inhibitor showed higher serum IL-10 levels than mice from the vehicle group. Serum levels of IL-4 and IL-5 Th2 cytokines tended to be higher in Ox-AD mice. However, no significant difference was evident between the $11 \beta$-HSD1 inhibitortreated and the vehicle-treated groups.

Th2 cells are increased in the skin of mice treated with 11ß-HSD1 inhibitor. We compared the percentage of regulatory $\mathrm{T}$ (Treg) cells that expressed foxp3 and CD25 among CD4-gated cells, and the percentage of Th2 cells that expressed IL-4 among CD3 and CD4-gated cells. The percentages of Treg cells and Th2 cells among splenic cells did not significantly differ between mice treated with the $11 \beta$-HSD1 inhibitor and the vehicle. The percentage of Th2 cells in skin cells tended to be higher in the $11 \beta$-HSD1 inhibitor-treated mouse group, although not significantly. Skin Treg cells did not show any significant difference between the two groups (Supplementary Fig. 1). 

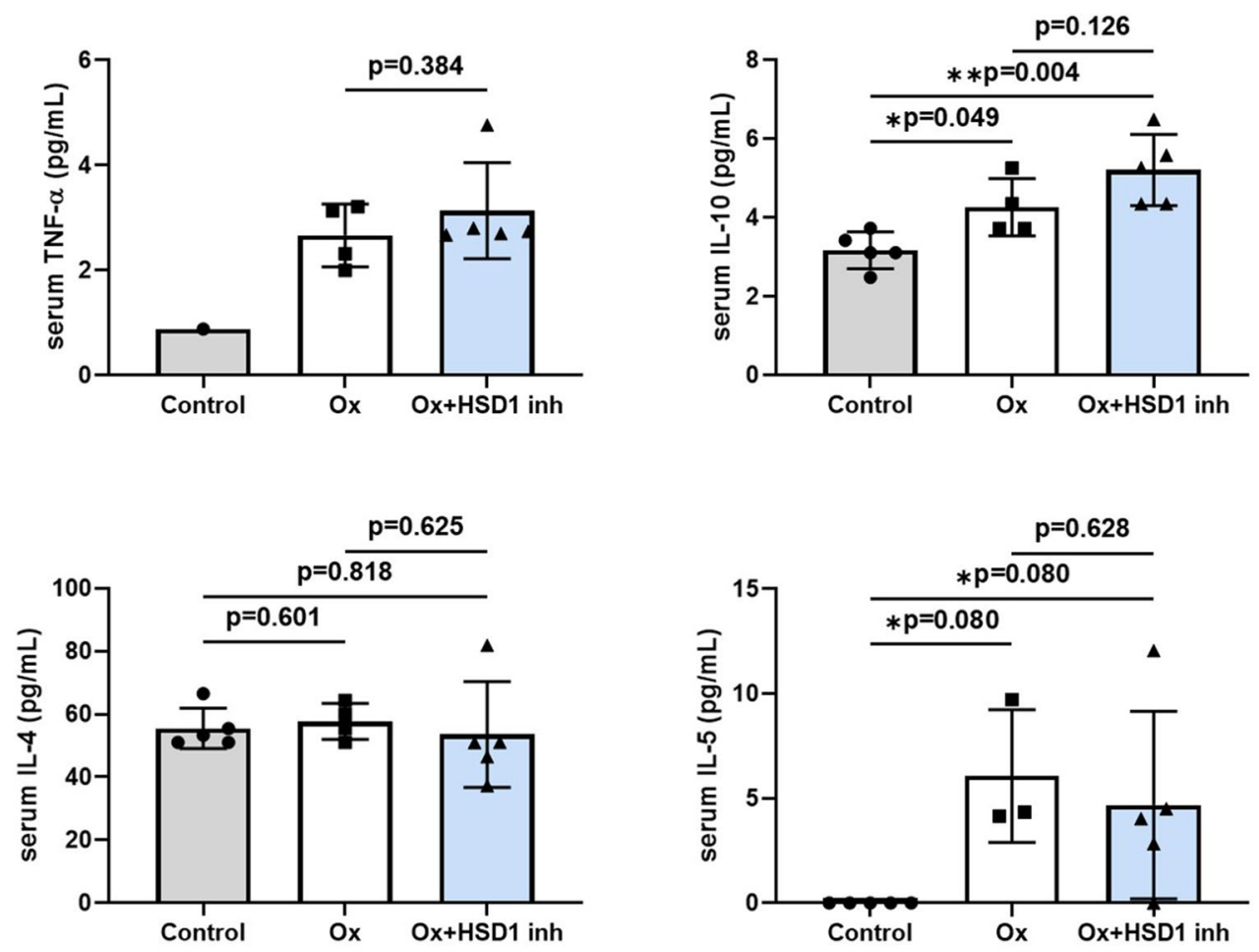

Figure 3. Serum inflammatory cytokines tend to be elevated in Ox-AD mice. Serum TNF- $\alpha$, IL-10, IL-4 and IL- 5 values determined by ELISA in control and Ox-induced mice with vehicle and $11 \beta$-HSD1 inhibitor $\left({ }^{*} p<0.05\right)$. Data are presented as means \pm standard deviation. HSD1 inh $11 \beta$-HSD1 inhibitor, Ox oxazolone.

(a)
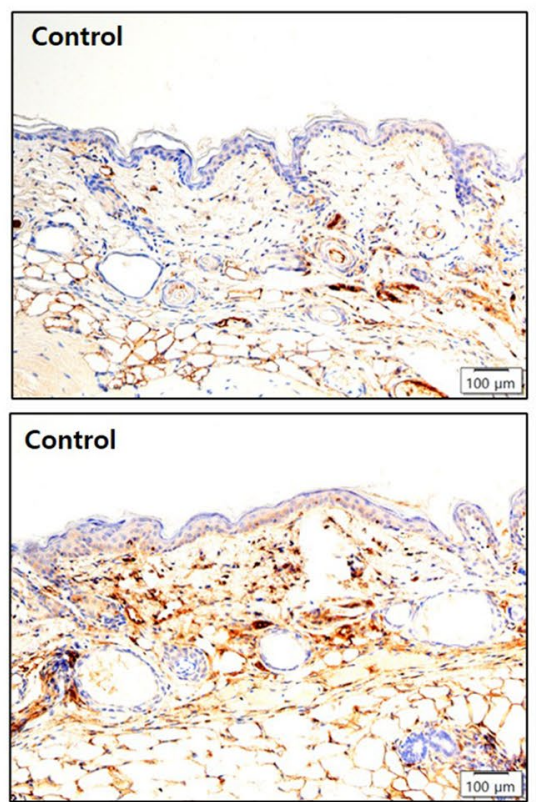
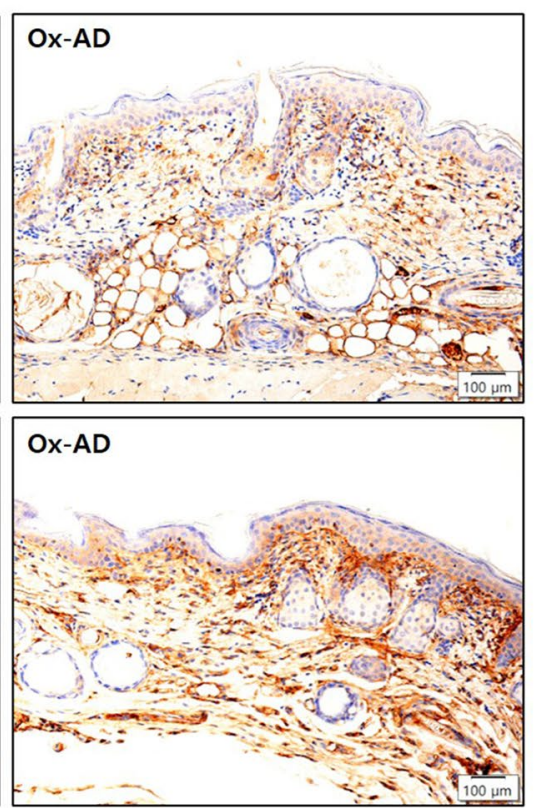

(b)

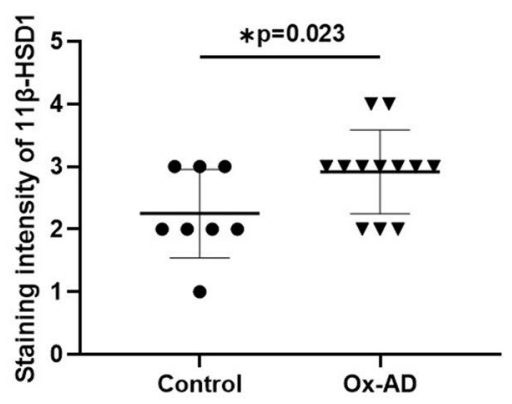

Figure 4. Epidermal 11 $\beta$-HSD1 expression is significantly increased in Ox-AD mice. (a) Immunohistochemical staining and (b) staining intensity of $11 \beta-H S D 1$ in the epidermis of control and Ox-AD mice (scale

bar $=100 \mu \mathrm{m}$ ). Staining intensity of $11 \beta$-HSD1 in the epidermis was graded as 0 (none), 1 (very weak), 2 (weak), 3 (moderate), 4 (strong), and 5 (very strong). Mean scores were compared between controls and Ox-AD mice $\left({ }^{*} p<0.05\right)$. Data are presented as means \pm standard deviation. $O x-A D$ oxazolone-induced atopic dermatitis. 
(a)

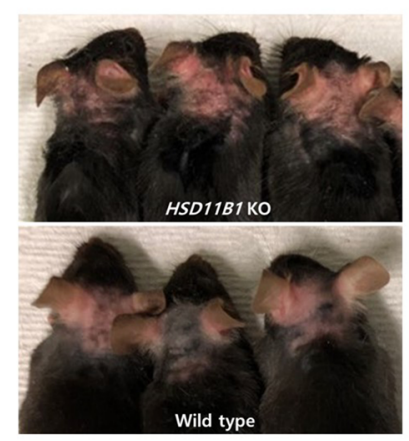

(d)

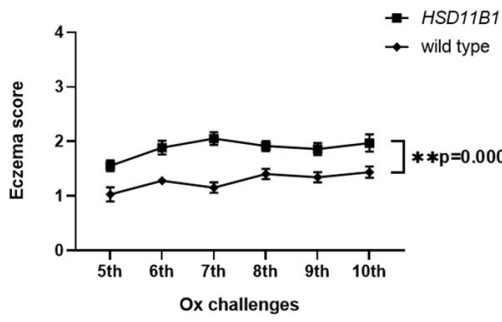

(b)

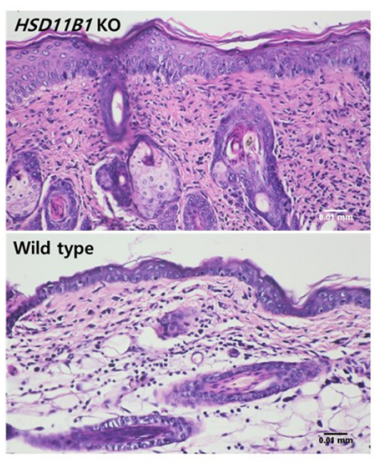

(e) (c)
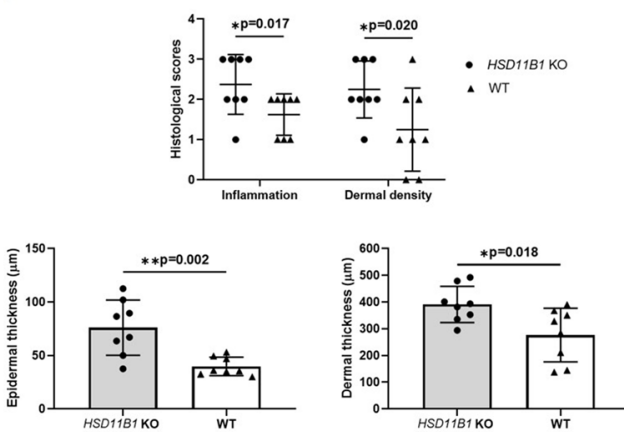

Figure 5. Oxazolone-induced atopic dermatitis in HSD11B1 KO and WT mice. (a) Gross photographs of skin lesions in global HSD11B1 KO mice with AD induced by Ox and WT mice ( $\mathrm{n}=8$ /group) taken before sacrifice (at 26 days). (b) Macroscopic appearance of skin sections of dorsal lesional surface. Scale bar, $10 \mu \mathrm{m}$. (c) Histological scores for dermal inflammation and density measured as epidermal and dermal thickness. (d) Eczema scores for HSD11B1 KO and WT mice at challenges 5-10. (e) TEWL, SC pH and SC hydration measured before sacrifice (at 26th day). (f) Corticosterone levels in epidermis of KO and WT mice measured by ELISA $\left({ }^{*} p<0.05,{ }^{* *} p<0.005\right)$. Data are presented as means \pm standard deviation. KO knock out, $S C$ stratum corneum, TEWL transepidermal water loss.

Epidermal 11ß-HSD1 expression is significantly increased in Ox-AD mice. The staining intensity of $11 \beta$-HSD1 was significantly higher in the epidermis of Ox-AD mice than in control mice (Fig. $4 \mathrm{a})$. Semiquantitative IHC scores (from 0 to 5$)$ of Ox-AD mice were also significantly higher than control mice $(p=0.023$; Fig. 4b).

Oxazolone worsened eczema in HSD11B1 KO mice compared to that in WT mice. Skin lesions were more severe after $10 \mathrm{Ox}$ challenges in mice with global HSD11B1 KO than in WT mice (Fig. 5a). The pathological features of both Ox-treated groups were characterized by marked epidermal hyperplasia with spongiosis, cellular infiltrates of mononuclear and polymorphonuclear cells, and dermal thickening, which is characteristic of $\mathrm{AD}$ (Fig. 5b). However, atopic inflammation was worse in $\mathrm{KO}$ mice due to the epidermis and dermis being significantly thickened. Histological scores for dermal inflammation and collagen density were also significantly higher in the KO than in the WT mice (Fig. 5c). Eczema scores were significantly higher in the HSD11B1 KO mice (Fig. 5d). The TEWL and pH of the SC were significantly higher in the HSD11B1 KO than in the WT mice, but SC hydration did not significantly differ between the groups (Fig. 5e). Corticosterone levels were higher in the KO than in the WT mice, although the difference was not significant (Fig. 5f).

Inflammatory cytokine expression is increased in skin of HSD11B1 KO mice. The mRNA expression levels of TSLP, IL-4, and IL-10, which are crucial cytokines in AD, were increased in the epidermis of KO mice compared to the expression levels in WT mice. IL-4 and IL-10 levels were significantly higher in the KO group ( $p=0.017$ and 0.013 , respectively). The mRNA expression of interferon-gamma (IFN- $\gamma$ ), an immunomodulatory cytokine, was also increased in $\mathrm{KO}$ mice, but the difference did not reach statistical significance (Fig. 6).

Epidermal 11 $\beta$-HSD1 expression is decreased in the lesional skin of patients with AD. The staining intensity of $11 \beta$-HSD1 was significantly lower in the epidermis of skin lesions of patients with AD than in non-lesional skin and healthy control skin (Fig. 7a). Semi-quantitative IHC scores showed no significant difference between control and non-lesional skin of patients with AD. Lesions of patients with AD tended to have lower staining intensity than skin of healthy controls and non-lesions of patients with $\mathrm{AD}$, but failed to show statistical significance (Fig. 7b). 

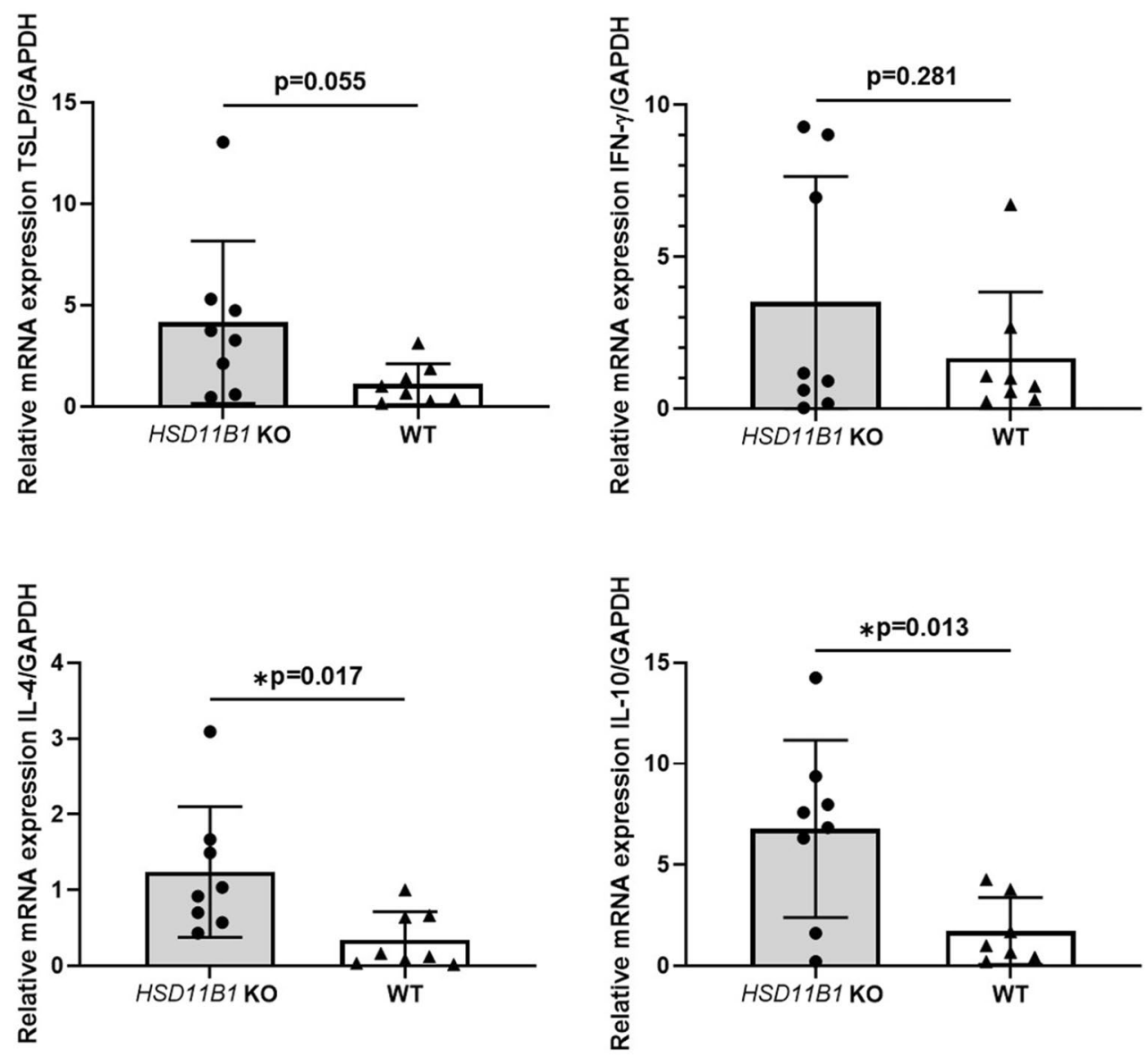

Figure 6. Increased inflammatory cytokine expression in skin of HSD11B1 KO mice. Expression of TSLP, IFN- $\gamma$, IL- 4 and IL-10 in epidermis of HSD11B1 KO and WT mice analyzed using qPCR $\left({ }^{*} p<0.05\right)$. Data are presented as means \pm standard deviation. KO, knockout; WT, wild type.

(a)

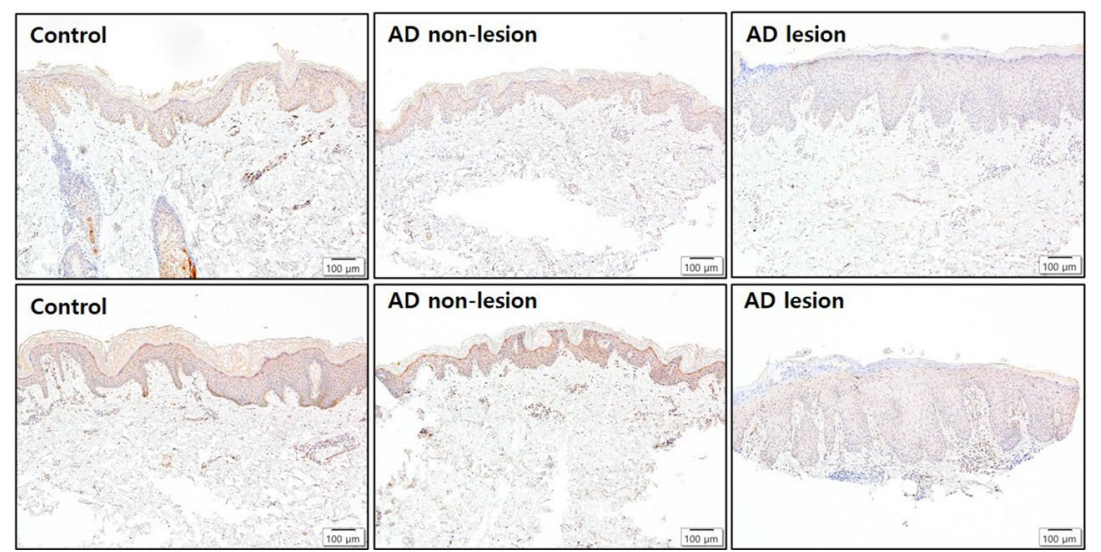

(b)

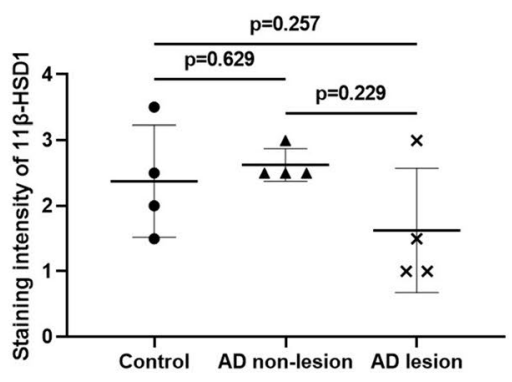

Figure 7. Epidermal $11 \beta$-HSD1 expression in the stratum corneum decreased in the lesional skin of patients with AD. (a) Immunohistochemical staining and (b) staining intensity of $11 \beta$-HSD1 in epidermis of lesions and non-lesions of four patients with $\mathrm{AD}$ and four individuals without $\mathrm{AD}$ (scale bar $=100 \mu \mathrm{m})$. Staining intensity of $11 \beta$-HSD1 in the epidermis was graded as 0 (none), 1 (very weak), 2 (weak), 3 (moderate), 4 (strong), and 5 (very strong). Mean scores were compared between controls, lesions, and non-lesions of patients with AD. Data are presented as means \pm standard deviation. $A D$ atopic dermatitis. 


\section{Discussion}

Human skin functions as an independent steroidogenic organ that maintains homeostasis against external stress $^{35,36}$. The role of $11 \beta$-HSD1 in inflammation has been studied. Inhibition of $11 \beta$-HSD1 in murine macrophages treated with lipopolysaccharide suppresses pro-inflammatory cytokine expression ${ }^{37}$. Moreover, inhibiting $11 \beta$-HSD1 reduces the TNFa-induced expression of IL-6, whereas $11 \beta$-HSD1 overexpression further augments IL-6 expression ${ }^{38}$. Knockdown of $11 \beta$-HSD1 in NHEK by siRNA transfection abrogates the induction of IL- 6 and IL- 8 by IL- $1 \beta$ and TNF $\alpha^{4}$. These results suggest that $11 \beta-H S D 1$ expression is needed to produce inflammatory cytokines.

On the contrary, $11 \beta$-HSD1 plays a protective role in models of inflammation in vivo. 11 $\beta$-HSD1-deficient $\mathrm{K} / \mathrm{B} \times \mathrm{N}$ serum-induced arthritis model mice showed earlier onset and slower resolution of inflammation ${ }^{39}$. When contact dermatitis was induced in mice using hapten, keratinocyte-specific $11 \beta$-HSD1 KO mice showed enhanced inflammation compared to WT mice ${ }^{24}$. These inconsistent results may reflect the complexity of systemic and local GC production affected by a de novo pathway and $11 \beta-\mathrm{HSD} 1$ action.

Two distinct $11 \beta$-HSDs and four distinct $17 \beta$-HSDs are known in mammals. The preference of the two $11 \beta$-HSDs and four $17 \beta$-HSDs for catalyzing oxidation or reduction of substrates has physiological benefits. According to Baker et al., the selective expression of different enzymes at different times in different tissues can control the concentration of active GCs or estrogens and androgens, providing a mechanism for regulating the actions of these hormones and maintaining normal physiology ${ }^{40}$. Phylogeny suggests that evolution of these enzymes from ancestor HSD may have occurred during the transition of vertebrates from ocean to land due to selective pressures from the colonization of land. $11 \beta-$ HSD 1 is most abundant in the liver and adipose tissue, but it is also widely expressed in the epidermis and dermis. In contrast, 11 $\beta$-HSD2 shows lower expression in the $\operatorname{skin}^{41}$. As the skin is the outermost organ of the body and is in constant contact with the environment, inflammation frequently occurs and keratinocytes differentiate repeatedly. Due to the nature of the skin, $11 \beta$-HSD1 was highly conserved in the skin through human evolution and served as an "endogenous pharmacy." However, complications, such as skin aging and inhibition of wound healing, appear when the enzyme is overexpressed as a result of UV exposure and endogenous aging.

We found that $11 \beta$-HSD1 expression was increased in the epidermis of flaky tail mouse models of congenital $\mathrm{AD}$ that spontaneously develop AD lesions. Sensitizing the skin of flaky tail mice with a mite allergen further increased $11 \beta$-HSD1 expression (data not published). Thus, we concluded that $11 \beta$-HSD1 action is important for dampening inflammation in the skin through GC activation, and that the reduced activity of this enzyme might promote the development of inflammatory skin diseases, including AD. Therefore, we speculated that inhibition of $11 \beta$-HSD1 decreases the levels of active cortisol in the skin and aggravates AD severity in the mouse model of Ox-induced AD.

A study to determine whether $11 \beta$-HSD1 knockdown in keratinocytes affects the production of TSLP that functions as a master switch for the Th2 immune response in $\mathrm{AD}^{42}$ found significantly higher TSLP production after stimulation with poly I:C and IL-4 in siRNA-transfected NHEK. These results agree with later findings ${ }^{33}$. Irradiation with UVB induces TSLP expression in human keratinocytes ${ }^{34}$. The present study found that siRNA transfection increased the amount of TSLP induced by UVB irradiation. Although we found that siRNA transfection almost completely inhibited $11 \beta$-HSD1 expression, $11 \beta-H S D 1 \mathrm{KO}$ only partly inhibited cortisol production by keratinocytes (Fig. 2b). This is because the skin can synthesize extra-adrenal glucocorticoid. Slominski et al. found that skin and skin cells express the genes and proteins required to activate the cytochrome P450scc system, indicating that skin can metabolize glucocorticoid from cholesterol ${ }^{43}$.

The local inhibition of $11 \beta$-HSD1 in the skin of Ox-AD hairless mice should aggravate the severity of atopic inflammation by suppressing GC activation. Eczema lesions, developed in the skin of hairless mice after four applications of $\mathrm{Ox}$ and worsened according to eczema scores in mice that were topically treated with an $11 \beta$ HSD1 inhibitor. The skin permeability barrier function, indicated by basal TEWL and SC hydration, was disrupted in $\mathrm{Ox}-\mathrm{AD}$ mice compared with that in control mice, but those treated with vehicle and with the 11 $\beta$-HSD1 inhibitor did not significantly differ.

There are no reported protocols regarding the concentration of topical 11 $\beta$-HSD1 inhibitor (385581). However, in a report published in 2014, Terao and colleagues dissolved 385581 in DMSO $(10 \mu \mathrm{M})$ for subcutaneous injection daily for 2 days $^{44}$. They also used $385581(50 \mu \mathrm{M})$ dissolved in acetone:olive oil (1:1) applied topically every day for 5 days and 385581 dissolved in PBS $(10 \mu \mathrm{M})$ to apply to skin wounds in a report published in $2011^{18}$. Neither report indicated a specific reference or consensus for their concentration choices. In our study, we chose a concentration of $100 \mu \mathrm{M}$ of 385581 dissolved in DMSO, which is easily accessible as a vehicle in the laboratory, and $100 \mu \mathrm{L}$ was applied to the skin twice a day. The results of our experiment were as expected from our hypothesis, suggesting that this concentration was effective.

Serum levels of inflammatory cytokines were higher in Ox-AD mice than in control mice, and even more so in mice with localized $11 \beta$-HSD1-inhibition, although the differences did not reach statistical significance. IL-10 induces Th2 but also has anti-inflammatory effects, and is increased in skin lesions in patients with $\mathrm{AD}^{45}$. Decreased IL-10 levels are associated with AD flares ${ }^{46}$. IL-10 forms heterodimers and binds to the IL-10 receptor to activate the Janus kinase-signal transducer and activator of transcription signaling pathway, notably Janus kinase 1, tyrosine kinase 2, and signal transducer and activator of transcription 3 and $1^{47}$. Its anti-inflammatory effects are mediated by inhibition of IFN- $\gamma$ and IL- 2 production by Th1 cells and inhibition of IL- 4 and IL-5 production by Th 2 cells through interference with B7/CD28-dependent signals ${ }^{48}$. The present study showed increased IL-10 levels in Ox-AD mice and higher mean IL-10 levels in mice that were topically treated with $11 \beta$-HSD1 inhibitor compared to vehicle-treated mice; however, the difference was not statistically significant. These results implied that although locally inhibited GC activation worsened eczema, it was insufficient to cause systemic effects. On the other hand, compared to WT mice, HSD11B1 KO mice had significantly higher levels of 
IL-10 in the skin. It would have been useful if genes or proteins associated with IL-10 were evaluated to determine the consequences of increased IL-10 on AD inflammation, but this could not be done due to a lack of samples.

Fluorescence-activated cell sorting (FACS) showed that the number of Th2 cells tended to increase in the skin of mice with localized $11 \beta$-HSD1 inhibition. However, the numbers of foxp $3+$ Treg and splenic cells in skin did not significantly differ. Treg cells are immuneregulators that diminish excessive immune responses, including those of harmful Th2 cell ${ }^{49}$. Because splenic cells represent systemic inflammation, topical 11 $\beta$-HSD1 inhibition might have weakened Th2 inflammation exclusively in the skin by blocking GC conversion to its active form. This may share meaning with the cytokine analysis in the skin, although we could not measure cytokine levels in the skin of mice due to a lack of skin samples.

We assessed the impact of systemic $11 \beta-\mathrm{HSD} 1$ inhibition on the emergence of AD lesions in global HSD11B1 KO mice in vivo. Others have validated the role of $11 \beta-\mathrm{HSD} 1$ in KO mice. One study describes the phenotypes of mice with targeted $11 \beta$-HSD1 and $11 \beta$-HSD2 deletions. Serum corticosterone is hypersecreted and the adrenal glands are hypertrophied in HSD11B1 KO mice due to the absence of normal negative feedback and failure of the HPA axis to compensate $^{50}$. Fasting glucose levels were similar between the KO and WT mice, but the responses of gluconeogenic enzymes were attenuated after starvation in the $\mathrm{KO}$ mice and they were resistant to hyperglycemia when stressed. Others have noted a discrepancy between secretion of local and systemic corticosterone in these KO mice, which has hindered clarification of the role of $11 \beta$-HSD1 in dermal collagen metabolism ${ }^{44}$. In contrast, one study found that HPA axis abnormalities in response to $11 \beta$-HSD1 are strain-dependent ${ }^{51}$, because congenic HSD1 KO mice on a C57Bl/6J background have normal basal plasma corticosterone and ACTH concentrations, in contrast to 129/MF1 mice that are null for $11 \beta-H S D 1$. Others have found that age-induced dermal atrophy is reversed and that the collagen biosynthesis gene is expressed in $H S D 11 B 1 \mathrm{KO}$ mice ${ }^{23}$. Results obtained with keratinocyte-specific HSD11B1 $\mathrm{KO}$ mice were consistent with the notion that corticosterone activation by $11 \beta-\mathrm{HSD} 1$ in keratinocytes represses local inflammation ${ }^{22,24}$; this rules out the impact of a compensatory increase in serum corticosterone in global $\mathrm{KO}$ mice. Moreover, serum corticosterone concentrations did not differ between WT and keratinocyte-specific KO mice.

We confirmed that the systemic inhibition of $11 \beta$-HSD1 significantly worsened AD evoked by Ox. These results were also supported by the histological features of a thickened epidermis and dermis, as well as higher scores for dermal inflammation and density in $\mathrm{KO}$ mice. Skin barrier permeability functions were also decreased in the KO mice. TEWL and SC pH was higher in KO mice. Elevated SC pH increases activity of kallikreins, which contributes to over degradation of corneodesmosomes followed by weakening of SC integrity and cohesion. It also impairs lipid processing and disrupts the permeability barrier of the epidermis. Therefore, antigen uptake increases through the disrupted skin barrier, which activates Langerhans cells, and then PAR-2 and TSLP are increased in the blood as well as the skin, contributing to AD-like inflammation ${ }^{52,53}$.

The principle cytokines involved in the pathogenesis of $\mathrm{AD}$ were increased in the epidermis of $\mathrm{KO}$ mice compared with WT mice. In contrast, corticosterone levels tended to be higher in the epidermis of KO mice. This might have been due to excessive corticosterone production by the central HPA axis or local production by the peripheral HPA axis in the skin to compensate for insufficient peripheral corticosterone. We investigated whether HSD11B1 KO mice have functional circadian rhythms by measuring serum corticosterone ( $\mathrm{n}=1$ per time point). Corticosterone values peaked at 1 the 5:00 and 18:30 hours and the reached at rough at 09:00 and 21:00 hours. The overall trend was similar to previous findings but differed somewhat in terms of peak timing (Supplementary Fig. 3). The range of serum corticosterone values was similar to that of previous findings ${ }^{54}$. We collected blood and skin samples at 09:00 hours which could be a disadvantage, but four investigators simultaneously sampled the mice, therefore it could be assumed that results were not influenced by the difference in time of sample collection.

The intensity of IHC staining for $11 \beta$-HSD1 in the epidermis showed the opposite results between murine AD models and human subjects. AD mice induced with Ox application exhibited an increased concentration of $11 \beta$ HSD1 in the epidermis compared to the controls. On the contrary, lesional skin of patients with AD showed the lowest $11 \beta$-HSD1 intensity compared to non-lesions of patients with $\mathrm{AD}$ and healthy control subjects, although the $p$-value did not reach statistical significance. Our results are similar to those of Terao et al., who reported that the expression of $11 \beta$-HSD1 increased in the epidermis of murine models after exposure of the models to $0.2 \%$ $\mathrm{Oxa}^{24}$; however, the expression of $11 \beta$-HSD1 is significantly decreased in the epidermis of patients with $\mathrm{AD}^{33}$. The discrepancy between murine and human subjects has yet to be investigated, but we propose the possibility that although short-term inflammation enhances $11 \beta$-HSD1 levels to suppress the inflammation, consistent longterm production of Th2 type cytokines may exert an inhibitory effect on the activity of $11 \beta$-HSD1 in the skin.

Our study results in vitro and in vivo confirmed that $11 \beta-H S D 1$ plays a role in the development of AD. As a pathogenic mechanism of $\mathrm{AD}$, disruption of the skin permeability barrier leads to the increase of various inflammatory cytokine, evoking chronic inflammation in the skin ${ }^{14,55}$. In addition, activation of $11 \beta$-HSD 1 in the skin protects against atopic inflammation by increasing the local availability of active GC, which are potent immuno-suppressants. Although control of systemic cortisol/corticosterone by the HPA axis is important for anti-inflammation, the de novo pathway and local control by $11 \beta$-HSD1 are also important in the skin. We have identified that inhibition of $11 \beta$-HSD1 activity alone aggravates AD inflammation irrespective of serum CS levels, and also verified that $11 \beta$-HSD1 activity is decreased in the skin of AD patients. In conclusion, insufficient $11 \beta$ HSD1 production or activity could function in the intricate pathophysiology of AD. If so, using methods that control local activity of $11 \beta$-HSD1 in the skin to treat atopic dermatitis could provide further insight into novel therapeutic approaches that prevent side effects from steroids that control systemic corticosterone.

\section{Methods}

Studies in vitro. Transfection with siRNA. We transfected NHEKs with $50 \mathrm{nM} 11 \beta$-HSD1 siRNA or control siRNA (Bioneer, Daejeon, Korea) using a mixture of Opti-MEM and Lipofectamine RNAiMAX reagent (Invitrogen, Carlsbad, CA, USA). The culture medium was replaced after six hours and the cells were used for 
experiments at $48 \mathrm{~h}$ after transfection. Detailed information about siRNA transfection, poly I:C and IL-4 treatment, UVB irradiation, qPCR, and ELISA is provided in the online Supplementary Materials and Methods.

Studies in vivo. Mouse experiments. The Institutional Animal Care and Use Committee at Yonsei University Wonju College of Medicine approved all animal procedures (YWC-150303-1). All animal handling procedures and human involvement methods were carried out in accordance with relevant guidelines and regulations. Eight-week-old female hairless mice $(\mathrm{hr} / \mathrm{hr}$ ) were obtained from OrientBio (Seongnam, Korea). Oxazolone was purchased from Sigma-Aldrich Co. (St. Louis, MO, USA). The selective 11 $\beta$-HSD1 inhibitor, 385581 (Merck \& Co., Kenilworth, NJ, USA), was dissolved in dimethyl sulfoxide (DMSO; $100 \mu \mathrm{M}$ ) vehicle for topical application.

The control group consisted of five mice, and the Ox-treated groups consisted of seven mice, each. Atopic dermatitis-like lesions were induced by the topical application of Ox (Supplementary Fig. 2). The mice were sensitized with a single application of $1 \% \mathrm{Ox}(60 \mu \mathrm{L})$. One week later, $0.1 \% \mathrm{Ox}(60 \mu \mathrm{L})$ was topically applied every other day for 20 days. We concurrently applied $100 \mu \mathrm{L}$ of 385581 twice daily to both sides of half of the mice (seven) with induced AD and DMSO vehicle to the other half (seven mice). The control group was similarly treated with acetone. Eczema was scored at every challenge, and gross AD lesions were photographed on the day the mice were sacrificed. The eczema score was defined as the average of individual scores for symptoms of erythema, edema, and lichenification, and graded as 0 (none), 1 (mild), 2 (moderate) or 3 (severe) ${ }^{56}$. Excoriation was excluded, as it is difficult to evaluate in mice. We measured TEWL on the dorsal skin of mice using a TEWA meter (Courage + Khazaka Electronics GmbH., Köln, Germany) and SC hydration using a Corneometer (Courage + Khazaka) before sacrifice (at 26 days). Dorsal skin, spleen and blood samples were collected from the mice post mortem.

Histological analysis. Detailed descriptions are provided in the Supplementary Materials and Methods, which are available online.

Enzyme-linked immunosorbent assay (ELISA). Detailed descriptions are provided in the online Supplementary Materials and Methods.

Flow cytometry. Detailed descriptions are provided in the online Supplementary Materials and Methods.

HSD11B1 KO mice. Mouse embryos with global HSD11B1 KO were supplied by Professor Gareth G. Lavery of the Molecular Metabolism Centre for Endocrinology, Diabetes, and Metabolism, School of Clinical and Experimental Medicine, Institute of Biomedical Research University of Birmingham, UK. We generated and injected HSD11B1 KO gene clones into C57BL/6 blastocysts as described ${ }^{57}$. The embryos were transferred into pseudopregnant C57BL/6 female mice and heterozygous mice were generated. Germline transmission of the $\mathrm{KO}$ allele was confirmed by PCR genotyping, then homozygous HSD11B1 KO mice were obtained after several breeding cycles Details of the genotyping are provided in the online Supplementary Materials and Methods.

Experiments using HSD11B1 KO mice. Oxazolone was applied as described above to induce AD-like lesions in 11 - to 20 -week-old KO and WT mice ( $\mathrm{n}=8$ each). One day after the $10^{\text {th }} \mathrm{Ox}$ challenge, eczema was scored and photographed in each mouse, then TEWL, SC hydration, and SC pH were measured before sacrifice at 26 days. Dorsal and ear skin, and blood samples were taken post mortem.

Histological analysis. Detailed descriptions are provided in the online Supplementary Materials and Methods.

Quantitative polymerase chain reaction ( $q P C R)$. A detailed description of the $\mathrm{qPCR}$ is provided in the online Supplementary Materials and Methods.

Studies on human samples. Detailed descriptions are provided in Supplementary Materials and Methods, which are available online.

Statistical analyses. Data in graphs are expressed as means \pm standard deviation. Differences between groups were analyzed by Student $t$ tests using GraphPad Prism (GraphPad Software, La Jolla, CA, USA). Differences between treated groups at each time point were determined using two-way analysis of variance (ANOVA) followed by Bonferroni-Dunn tests. Mann-Whitney test was used to compare the staining intensities of human skin samples. Values with $\mathrm{p}<0.05$ were considered statistically significant.

\section{Data availability}

No datasets were generated or analyzed during the current study.

Received: 31 July 2020; Accepted: 6 November 2020

Published online: 19 November 2020 


\section{References}

1. Nicolaides, N. C., Charmandari, E., Chrousos, G. P. \& Kino, T. Circadian endocrine rhythms: the hypothalamic-pituitary-adrenal axis and its actions. Ann. N. Y. Acad. Sci. 1318, 71-80. https://doi.org/10.1111/nyas.12464 (2014).

2. Adcock, I. M. \& Caramori, G. Cross-talk between pro-inflammatory transcription factors and glucocorticoids. Immunol. Cell Biol. 79, 376-384. https://doi.org/10.1046/j.1440-1711.2001.01025.x (2001).

3. Stojadinovic, O. et al. Novel genomic effects of glucocorticoids in epidermal keratinocytes: inhibition of apoptosis, interferongamma pathway, and wound healing along with promotion of terminal differentiation. J. Biol. Chem. 282, 4021-4034. https://doi. org/10.1074/jbc.M606262200 (2007).

4. Itoi, S., Terao, M., Murota, H. \& Katayama, I. 11beta-Hydroxysteroid dehydrogenase 1 contributes to the pro-inflammatory response of keratinocytes. Biochem. Biophys. Res. Commun. 440, 265-270. https://doi.org/10.1016/j.bbrc.2013.09.065 (2013).

5. Skobowiat, C., Dowdy, J. C., Sayre, R. M., Tuckey, R. C. \& Slominski, A. Cutaneous hypothalamic-pituitary-adrenal axis homolog: regulation by ultraviolet radiation. Am. J. Physiol. Endocrinol. Metab. 301, E484-E493. https://doi.org/10.1152/ajpendo.00217.2011 (2011).

6. Hannen, R. F. et al. Steroid synthesis by primary human keratinocytes; implications for skin disease. Biochem. Biophys. Res. Commun. 404, 62-67. https://doi.org/10.1016/j.bbrc.2010.11.059 (2011).

7. Cirillo, N. \& Prime, S. S. Keratinocytes synthesize and activate cortisol. J. Cell Biochem. 112, 1499-1505. https://doi.org/10.1002/ jcb.23081 (2011)

8. Slominski, A. \& Wortsman, J. Neuroendocrinology of the skin. Endocr. Rev. 21, 457-487. https://doi.org/10.1210/edrv.21.5.0410 (2000).

9. Taves, M. D., Gomez-Sanchez, C. E. \& Soma, K. K. Extra-adrenal glucocorticoids and mineralocorticoids: evidence for local synthesis, regulation, and function. Am. J. Physiol. Endocrinol. Metab. 301, E11-E24. https://doi.org/10.1152/ajpendo.00100.2011 (2011).

10. Slominski, A. T. et al. Cytochromes p450 and skin cancer: role of local endocrine pathways. Anticancer Agents Med. Chem. 14, 77-96 (2014).

11. Slominski, A. T. et al. Sensing the environment: regulation of local and global homeostasis by the skin's neuroendocrine system. Adv. Anat. Embryol. Cell Biol. 212, v, vii, 1-115 (2012).

12. Slominski, A. et al. CRH stimulation of corticosteroids production in melanocytes is mediated by ACTH. Am. J. Physiol. Endocrinol. Metab. 288, E701-E706. https://doi.org/10.1152/ajpendo.00519.2004 (2005).

13. Slominski, A., Zbytek, B., Szczesniewski, A. \& Wortsman, J. Cultured human dermal fibroblasts do produce cortisol. J. Investig. Dermatol. 126, 1177-1178. https://doi.org/10.1038/sj.jid.5700204 (2006).

14. Lin, T. K., Zhong, L. \& Santiago, J. L. Association between stress and the HPA axis in the atopic dermatitis. Int. J. Mol. Sci. https:// doi.org/10.3390/ijms18102131 (2017).

15. Cooper, M. S. \& Stewart, P. M. 11Beta-hydroxysteroid dehydrogenase type 1 and its role in the hypothalamus-pituitary-adrenal axis, metabolic syndrome, and inflammation. J. Clin. Endocrinol. Metab. 94, 4645-4654. https://doi.org/10.1210/jc.2009-1412 (2009).

16. Vukelic, S. et al. Cortisol synthesis in epidermis is induced by IL-1 and tissue injury. J. Biol. Chem. 286, 10265-10275. https://doi. org/10.1074/jbc.M110.188268 (2011).

17. Skobowiat, C., Sayre, R. M., Dowdy, J. C. \& Slominski, A. T. Ultraviolet radiation regulates cortisol activity in a waveband-dependent manner in human skin ex vivo. Br. J. Dermatol. 168, 595-601. https://doi.org/10.1111/bjd.12096 (2013).

18. Terao, M. et al. 11beta-Hydroxysteroid dehydrogenase-1 is a novel regulator of skin homeostasis and a candidate target for promoting tissue repair. PLoS ONE 6, e25039. https://doi.org/10.1371/journal.pone.0025039 (2011).

19. Tiganescu, A., Walker, E. A., Hardy, R. S., Mayes, A. E. \& Stewart, P. M. Localization, age- and site-dependent expression, and regulation of 11beta-hydroxysteroid dehydrogenase type 1 in skin. J. Investig. Dermatol. 131, 30-36. https://doi.org/10.1038/ jid.2010.257 (2011).

20. Miller, W. L. \& Auchus, R. J. The molecular biology, biochemistry, and physiology of human steroidogenesis and its disorders. Endocr. Rev. 32, 81-151. https://doi.org/10.1210/er.2010-0013 (2011).

21. Tiganescu, A. et al. UVB induces epidermal 11beta-hydroxysteroid dehydrogenase type 1 activity in vivo. Exp. Dermatol. 24, 370-376. https://doi.org/10.1111/exd.12682 (2015).

22. Itoi-Ochi, S., Terao, M., Murota, H. \& Katayama, I. Local corticosterone activation by 11 beta-hydroxysteroid dehydrogenase 1 in keratinocytes: the role in narrow-band UVB-induced dermatitis. Dermatoendocrinology 8, e1119958. https://doi.org/10.1080/19381 980.2015.1119958 (2016).

23. Tiganescu, A. et al. 11 beta-Hydroxysteroid dehydrogenase blockade prevents age-induced skin structure and function defects. J. Clin. Investig. 123, 3051-3060. https://doi.org/10.1172/JCI64162 (2013).

24. Terao, M. et al. Local glucocorticoid activation by 11 beta-hydroxysteroid dehydrogenase 1 in keratinocytes: the role in hapteninduced dermatitis. Am. J. Pathol. 186, 1499-1510. https://doi.org/10.1016/j.ajpath.2016.01.014 (2016).

25. Elias, P. M. \& Wakefield, J. S. Therapeutic implications of a barrier-based pathogenesis of atopic dermatitis. Clin. Rev. Allergy Immunol. 41, 282-295. https://doi.org/10.1007/s12016-010-8231-1 (2011).

26. Ohtsu, H. \& Seike, M. Histamine and histamine receptors in allergic dermatitis. Handb. Exp. Pharmacol. 241, 333-345. https:// doi.org/10.1007/164_2016_9 (2017).

27. Guttman-Yassky, E. \& Krueger, J. G. Atopic dermatitis and psoriasis: two different immune diseases or one spectrum?. Curr. Opin. Immunol. 48, 68-73. https://doi.org/10.1016/j.coi.2017.08.008 (2017).

28. Gittler, J. K. et al. Progressive activation of $\mathrm{T}(\mathrm{H}) 2 / \mathrm{T}(\mathrm{H}) 22$ cytokines and selective epidermal proteins characterizes acute and chronic atopic dermatitis. J. Allergy Clin. Immunol. 130, 1344-1354. https://doi.org/10.1016/j.jaci.2012.07.012 (2012).

29. Buske-Kirschbaum, A., Ebrecht, M. \& Hellhammer, D. H. Blunted HPA axis responsiveness to stress in atopic patients is associated with the acuity and severeness of allergic inflammation. Brain Behav. Immun. 24, 1347-1353. https://doi.org/10.1016/j. bbi.2010.06.013 (2010).

30. Buske-Kirschbaum, A., Geiben, A., Hollig, H., Morschhauser, E. \& Hellhammer, D. Altered responsiveness of the hypothalamuspituitary-adrenal axis and the sympathetic adrenomedullary system to stress in patients with atopic dermatitis. J. Clin. Endocrinol. Metab. 87, 4245-4251. https://doi.org/10.1210/jc.2001-010872 (2002).

31. Priftis, K. N., Papadimitriou, A., Nicolaidou, P. \& Chrousos, G. P. The hypothalamic-pituitary-adrenal axis in asthmatic children. Trends Endocrinol. Metab. 19, 32-38. https://doi.org/10.1016/j.tem.2007.10.005 (2008).

32. Sevilla, L. M., Latorre, V., Sanchis, A. \& Perez, P. Epidermal inactivation of the glucocorticoid receptor triggers skin barrier defects and cutaneous inflammation. J. Investig. Dermatol. 133, 361-370. https://doi.org/10.1038/jid.2012.281 (2013).

33. Matsumoto, A., Murota, H., Terao, M. \& Katayama, I. Attenuated activation of homeostatic glucocorticoid in keratinocytes induces alloknesis via aberrant artemin production. J. Investig. Dermatol. 138, 1491-1500. https://doi.org/10.1016/j.jid.2018.02.010 (2018).

34. Jang, Y. et al. UVB induces HIF-1alpha-dependent TSLP expression via the JNK and ERK pathways. J. Investig. Dermatol. 133, 2601-2608. https://doi.org/10.1038/jid.2013.203 (2013). 
35. Slominski, A. et al. Steroidogenesis in the skin: implications for local immune functions. J. Steroid Biochem. Mol. Biol. 137, 107-123. https://doi.org/10.1016/j.jsbmb.2013.02.006 (2013).

36. Slominski, A. T., Manna, P. R. \& Tuckey, R. C. On the role of skin in the regulation of local and systemic steroidogenic activities. Steroids 103, 72-88. https://doi.org/10.1016/j.steroids.2015.04.006 (2015).

37. Ishii, T. et al. Augmentation of 11 beta-hydroxysteroid dehydrogenase type 1 in LPS-activated J774.1 macrophages-role of 11betaHSD1 in pro-inflammatory properties in macrophages. FEBS Lett. 581, 349-354. https://doi.org/10.1016/j.febslet.2006.11.032 (2007).

38. Ishii-Yonemoto, T. et al. Glucocorticoid reamplification within cells intensifies NF-kappaB and MAPK signaling and reinforces inflammation in activated preadipocytes. Am. J. Physiol. Endocrinol. Metab. 298, E930-E940. https://doi.org/10.1152/ajpendo.00320 2009 (2010).

39. Coutinho, A. E. et al. 11beta-Hydroxysteroid dehydrogenase type 1, but not type 2, deficiency worsens acute inflammation and experimental arthritis in mice. Endocrinology 153, 234-240. https://doi.org/10.1210/en.2011-1398 (2012).

40. Baker, M. E. Unusual evolution of 11beta- and 17beta-hydroxysteroid and retinol dehydrogenases. BioEssays 18, 63-70. https:// doi.org/10.1002/bies.950180112 (1996).

41. Chapman, K., Holmes, M. \& Seckl, J. 11beta-hydroxysteroid dehydrogenases: intracellular gate-keepers of tissue glucocorticoid action. Physiol. Rev. 93, 1139-1206. https://doi.org/10.1152/physrev.00020.2012 (2013).

42. Cianferoni, A. \& Spergel, J. The importance of TSLP in allergic disease and its role as a potential therapeutic target. Expert Rev. Clin. Immunol. 10, 1463-1474. https://doi.org/10.1586/1744666X.2014.967684 (2014).

43. Slominski, A. et al. A novel pathway for sequential transformation of 7-dehydrocholesterol and expression of the P450scc system in mammalian skin. Eur. J. Biochem. 271, 4178-4188. https://doi.org/10.1111/j.1432-1033.2004.04356.x (2004).

44. Terao, M. et al. 11beta-hydroxysteroid dehydrogenase 1 specific inhibitor increased dermal collagen content and promotes fibroblast proliferation. PLoS ONE 9, e93051. https://doi.org/10.1371/journal.pone.0093051 (2014).

45. Ohmen, J. D. et al. Overexpression of IL-10 in atopic dermatitis. Contrasting cytokine patterns with delayed-type hypersensitivity reactions. J. Immunol. 154, 1956-1963 (1995).

46. Darling, A. R. et al. IL-10 suppresses IL-17-mediated dermal inflammation and reduces the systemic burden of Vaccinia virus in a mouse model of eczema vaccinatum. Clin. Immunol. 150, 153-160. https://doi.org/10.1016/j.clim.2013.11.010 (2014).

47. Ouyang, W., Rutz, S., Crellin, N. K., Valdez, P. A. \& Hymowitz, S. G. Regulation and functions of the IL-10 family of cytokines in inflammation and disease. Annu. Rev. Immunol. 29, 71-109. https://doi.org/10.1146/annurev-immunol-031210-101312 (2011).

48. Jung, B. G., Cho, S. J., Ko, J. H. \& Lee, B. J. Inhibitory effects of interleukin-10 plasmid DNA on the development of atopic dermatitis-like skin lesions in NC/Nga mice. J. Vet. Sci. 11, 213-220. https://doi.org/10.4142/jvs.2010.11.3.213 (2010).

49. Ochs, H. D., Ziegler, S. F. \& Torgerson, T. R. FOXP3 acts as a rheostat of the immune response. Immunol. Rev. 203, 156-164. https ://doi.org/10.1111/j.0105-2896.2005.00231.x (2005).

50. Holmes, M. C., Kotelevtsev, Y., Mullins, J. J. \& Seckl, J. R. Phenotypic analysis of mice bearing targeted deletions of 11 betahydroxysteroid dehydrogenases 1 and 2 genes. Mol. Cell Endocrinol. 171, 15-20 (2001).

51. Carter, R. N. et al. Hypothalamic-pituitary-adrenal axis abnormalities in response to deletion of 11beta-HSD1 is strain-dependent. J. Neuroendocrinol. 21, 879-887. https://doi.org/10.1111/j.1365-2826.2009.01899.x (2009).

52. Lee, H. J. et al. Acidification of stratum corneum prevents the progression from atopic dermatitis to respiratory allergy. Exp. Dermatol. 26, 66-72. https://doi.org/10.1111/exd.13144 (2017).

53. Hatano, Y. et al. Maintenance of an acidic stratum corneum prevents emergence of murine atopic dermatitis. J. Investig. Dermatol. 129, 1824-1835. https://doi.org/10.1038/jid.2008.444 (2009).

54. Veniant, M. M. et al. Time of the day for 11beta-HSD1 inhibition plays a role in improving glucose homeostasis in DIO mice. Diabetes Obes. Metab. 11, 109-117. https://doi.org/10.1111/j.1463-1326.2008.00911.x (2009).

55. Kabashima, K. New concept of the pathogenesis of atopic dermatitis: interplay among the barrier, allergy, and pruritus as a trinity. J. Dermatol. Sci. 70, 3-11. https://doi.org/10.1016/j.jdermsci.2013.02.001 (2013).

56. Yang, F. et al. Topical application of rapamycin ointment ameliorates Dermatophagoides farina body extract-induced atopic dermatitis in NC/Nga mice. Exp. Dermatol. 23, 568-572. https://doi.org/10.1111/exd.12463 (2014).

57. Semjonous, N. M. et al. Hexose-6-phosphate dehydrogenase contributes to skeletal muscle homeostasis independent of 11 betahydroxysteroid dehydrogenase type 1. Endocrinology 152, 93-102. https://doi.org/10.1210/en.2010-0957 (2011).

\section{Acknowledgements}

This work was supported by the National Research Foundation of Korea (NRF) grant funded by the Korea government (MEST) (NRF-2018R1A2B2005002). We are grateful to Editage (https://www.editage.co.kr) for English language editing.

\section{Author contributions}

N.R.L.: conceptualization, formal analysis, investigation, methodology, resources, validation, visualization, writing (original draft preparation, review and editing). B.J.K.: investigation. C.H.L.: investigation. Y.B.L.: investigation. S.L.: investigation, formal analysis. H.J.H.: investigation, resources, visualization. E.K.: investigation, resources. S.H.K.: investigation, validation. M.G.L.: resources, validation. S.E.L.: investigation, resources, validation, visualization. G.G.L.: resources. E.H.C.: conceptualization, funding acquisition, methodology, project administration, resources, supervision, validation, writing (review and editing).

\section{Competing interests}

The authors declare no competing interests.

\section{Additional information}

Supplementary information is available for this paper at https://doi.org/10.1038/s41598-020-77281-X.

Correspondence and requests for materials should be addressed to E.H.C.

Reprints and permissions information is available at www.nature.com/reprints.

Publisher's note Springer Nature remains neutral with regard to jurisdictional claims in published maps and institutional affiliations. 
(c) (i) Open Access This article is licensed under a Creative Commons Attribution 4.0 International cc) License, which permits use, sharing, adaptation, distribution and reproduction in any medium or format, as long as you give appropriate credit to the original author(s) and the source, provide a link to the Creative Commons licence, and indicate if changes were made. The images or other third party material in this article are included in the article's Creative Commons licence, unless indicated otherwise in a credit line to the material. If material is not included in the article's Creative Commons licence and your intended use is not permitted by statutory regulation or exceeds the permitted use, you will need to obtain permission directly from the copyright holder. To view a copy of this licence, visit http://creativecommons.org/licenses/by/4.0/.

(C) The Author(s) 2020 